\title{
Blood Flow of Bubbles Moving in Microchannels with Bifurcations
}

\author{
D. Bento ${ }^{1}$, S. Lopes $^{2}$, I. Maia ${ }^{3}$, A. I. Pereira ${ }^{4,5}$, C. S. Fernandes ${ }^{2}$, \\ J. M. Miranda ${ }^{1(凶)}$, and R. Lima ${ }^{6}$ \\ ${ }^{1}$ CEFT, Faculdade de Engenharia da Universidade do Porto (FEUP), \\ Rua Dr. Roberto Frias, 4200-465 Porto, Portugal \\ jmiranda@fe.up.pt \\ ${ }^{2}$ Polytechnic Institute of Bragança, ESTiG/IPB, C. Sta. Apolonia, \\ 5301-857 Bragança, Portugal \\ ${ }^{3}$ Instituto Superior Técnico, Universidade de Lisboa, Av. Rovisco Pais, \\ 1049-001 Lisbon, Portugal \\ ${ }^{4}$ Research Centre in Digitalization and Intelligent Robotics (CeDRI), Instituto \\ Politécnico de Bragança, C. Sta. Apolónia, 5301-857 Bragança, Portugal \\ 5 Algoritmi R \& D Centre, University of Minho, Campus de Gualtar, \\ 4710-057 Braga, Portugal \\ 6 MEtRiCS, Mechanical Engineering Department, University of Minho, Campus \\ de Azurém, 4800-058 Guimarães, Portugal
}

\begin{abstract}
The gas embolism is a well-known phenomenon. Previous studies have been performed to understand the formation, the behavior and the influence of air bubbles in microcirculation. This study aims to investigate the flow of bubbles in a microchannel network with bifurcations. For that purpose, a microchannel network was fabricated by soft lithography. The working fluids used were composed by sheep red blood cells (RBCs) suspended in dextran 40 and two different hematocrits were studied, $5 \%$ and $10 \%$. The in vitro blood flow was analyzed for a flow rate of $10 \mu \mathrm{l} / \mathrm{min}$, by using an inverted microscope and a high-speed camera. It was possible to visualize the formation of the bubbles and their behavior along the network. The results show that the passage of air bubbles influences the cells local concentration, since a higher concentration of cells was seen upstream to the bubble and lower concentrations downstream to the bubble.
\end{abstract}

Keywords: Blood flow - Gas embolism - Microcirculation - Bifurcations · Networks

\section{Introduction}

The presence of gas inside of a blood vessel is known as gas embolism. This phenomenon is most commonly due to medical errors and can lead to serious consequences to the patient, including death [1]. The origin of air inside of blood vessel can be: (1) exogenous, when air is injected in the blood vessel, resulting from medical procedures (e.g. surgeries [2, 3], hemodialysis [4] and embolotherapy [5]); (2) or endogenous, when gas bubbles form inside the bloodstream (e.g. heart valves [6, 7], 
scuba diving [8], space operations [9] and high altitude flights [10] incidents). Understanding the formation of bubbles, their behavior in the microcirculation, as well as their influence on the blood flow are the objectives of several studies already performed [11-13]. Bubbles in small vessels flow as tubular bubbles [5, 13, 14]; they can be trapped on bifurcations or in small branches [5] or dissolve in blood, depending on their size, composition and shape [11].

It was observed in a previous work [13] that bubbles disturb the RBCs distribution leading to regions of high and of low concentrations of RBCs. In the present work, this phenomenon is further explored by analyzing the effect of bubbles in the RBCs distribution in the blood flow in a microchannel network.

\section{Materials and Methods}

\subsection{Working Fluids and Network Geometry}

For the preparation of the working fluids, blood was collected from a healthy sheep using a tube containing ethylenediaminetetraacetic acid (EDTA) to prevent coagulation. The RBCs were then separated from blood samples by centrifugation and aspiration and suspended in Dextran $40(D x 40)$ to make the samples with hematocrits (Hct) of 5\% and $10 \%$. Detailed procedure can found elsewhere [13, 15]. The microfluidic device used in this study was produced in PDMS by a conventional softlithography technique from SU-8 molds (Microliquid, Spain). The geometry used to perform the microfluidic experiments is shown in Fig. 1. The geometry consists in two inlets, one for the fluid and the other for the air. In addition, there is a network of successive bifurcations and at the end there is an outlet.

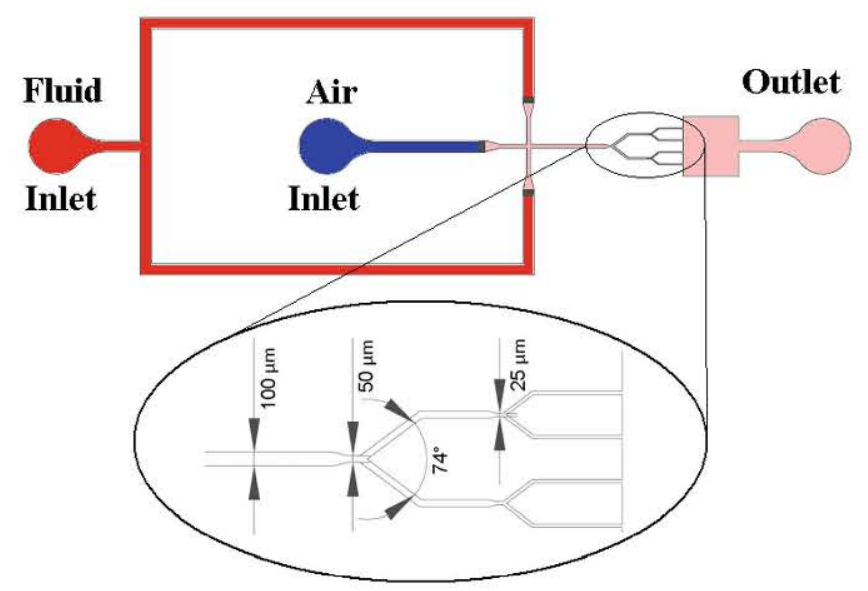

Fig. 1. Main dimensions of the geometry used in this study. 


\subsection{Experimental Set-Up}

The experimental set up comprises an inverted microscope (IX71, Olympus, Japan) combined with a high-speed camera (Fastcam SA3, Photron, USA). The PDMS microchannel was placed on the stage of the inverted microscope and a syringe pump (PHD ULTRA, Harvard Apparatus) was used to control the flow rate of the working fluids. The liquid flow rate was set to $10 \mu \mathrm{L} / \mathrm{min}$. Additionally, the air pressure was controlled by a pressure pump (Eleveflow PG1113). By adjusting the pressure, the pump has injected air droplets in the microchannel.

\subsection{Image Processing}

The images were recorded at the midplane of the microchannel using a frame rate of 2000 frames/second. All the frames were analyzed using the tool Plot $z$-axis profile of the ImageJ software. This function allows, after selecting a particular area of the video, to obtain a diagram with the tonality in the region of interest through time. By using this methodology, it was possible to evaluate the passage air bubbles in a region of interest (ROI) for a certain time.

\section{Results and Discussion}

In Fig. 2 we can see the formation of a bubble in the microchannel. Figure 2(a) shows the bubble before detachment whereas Fig. 2(b) shows the bubble after detachment.

a)

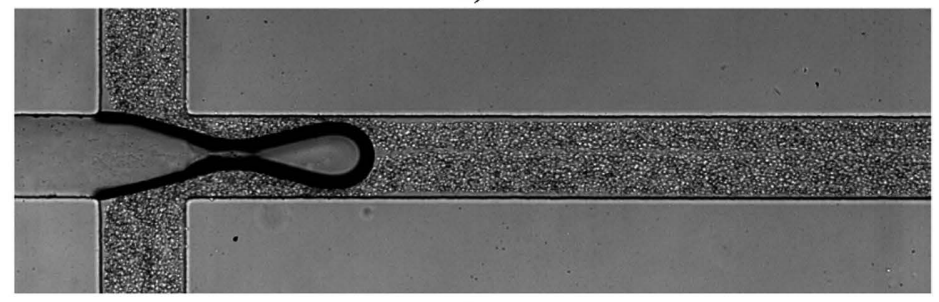

b)

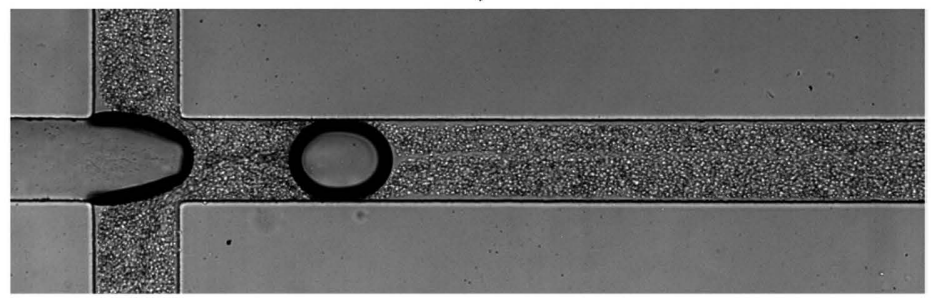

Fig. 2. Sequential images of the formation of a bubble air (a) bubble before detachment (b) bubble after detachment. 
The obtained videos were, firstly, carefully examined. It was observed that circulating air bubbles influence the local haematocrit located downstream and upstream to the bubble. Figure 3 shows this phenomenon at two different instants.
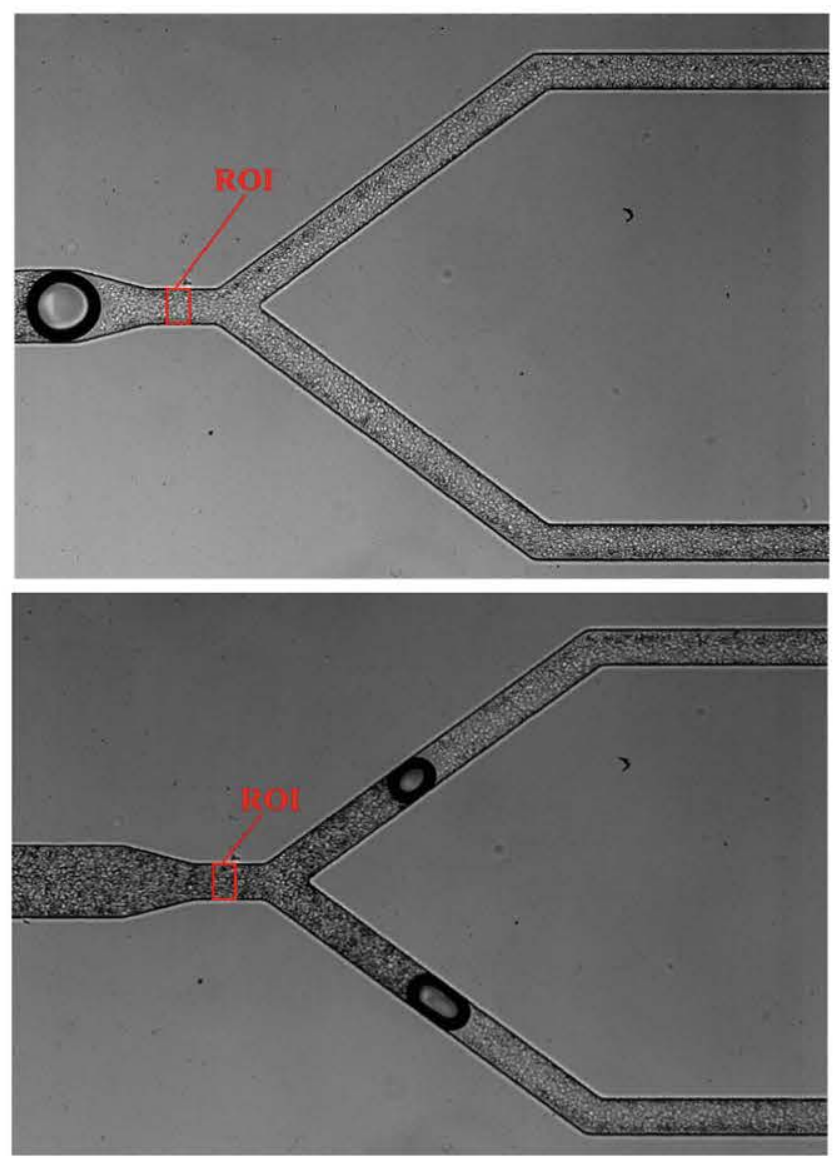

Fig. 3. Influence of air bubbles on the local hematocrit for a flow rate of $10 \mu 1 / \mathrm{min}$ and $10 \%$ Het.

By using the Plot z-axis profile tool from ImageJ, we were able to investigate the influence of the circulating bubbles on the local Hct. As shown in Fig. 4, it was possible to examine the variation of the Hct along a certain time, in a selected region (ROI) of the microchannel. The variation of the local Hct was analyzed by measuring the intensity of the pixels at the region where the plot Z-axis profile function was applied.

When the intensity of the pixels is constant, it means that the hematocrit that passes through this region is constant. If the intensity goes up, it indicates a decrease of the hematocrit at that region of interest (ROI). It is also possible to observe a drastic 
a)

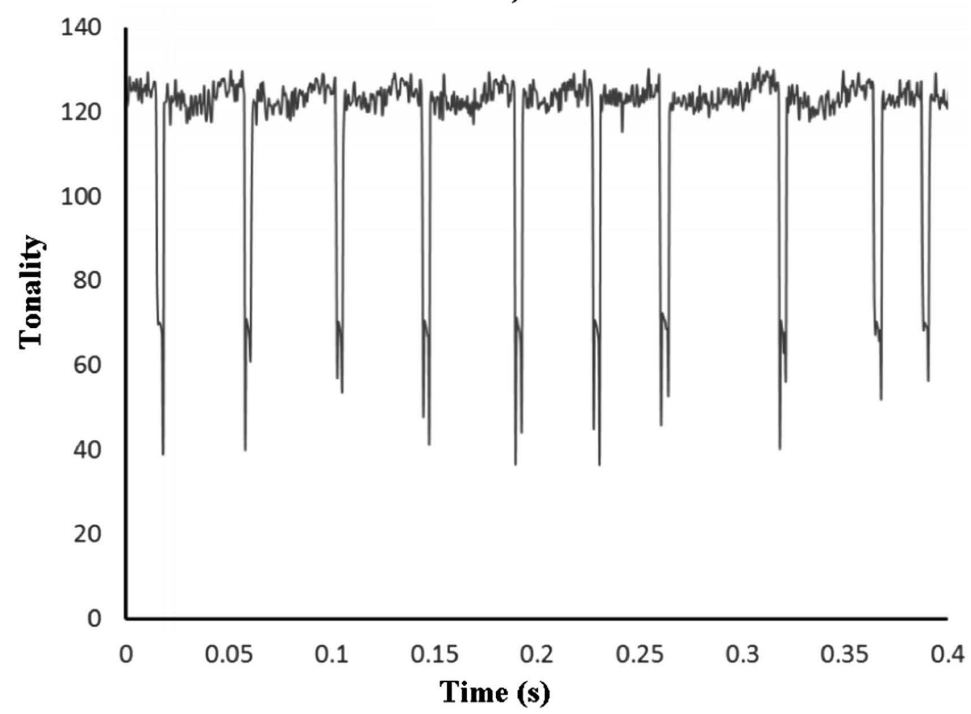

b)

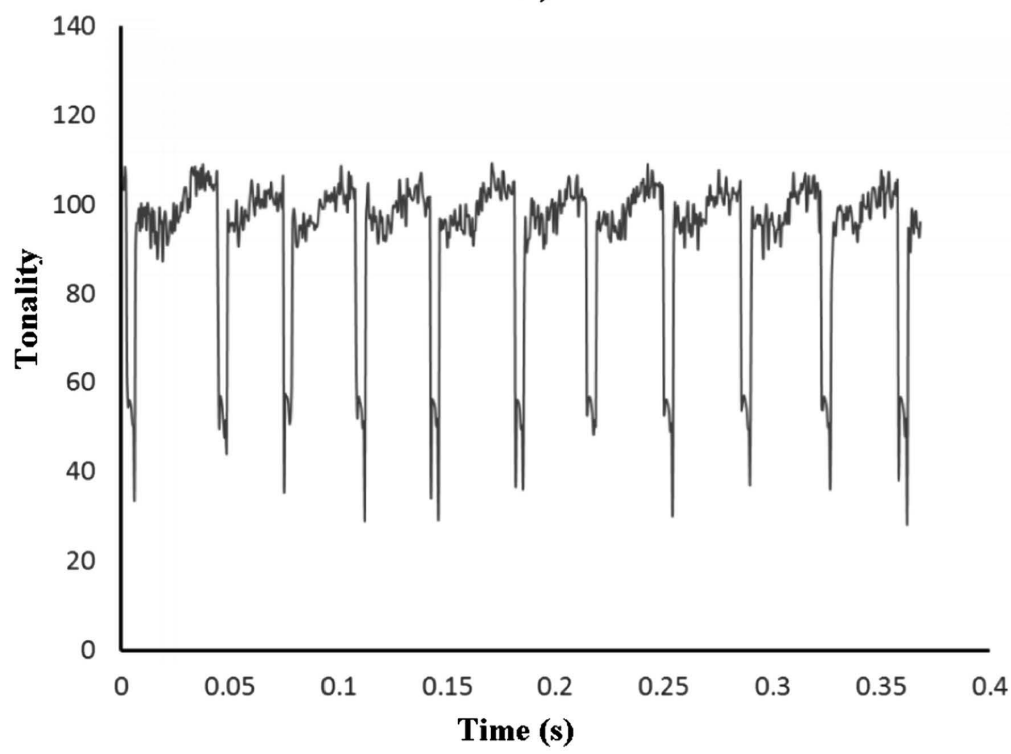

Fig. 4. Influence of air bubbles on the local Hct for a flow rate of $10 \mu 1 / \mathrm{min}$ and two different feed Hcts: (a) $5 \%$ Hct and (b) $10 \%$ Hct.

reduction of the tonality that indicates the passage of the air bubbles at the ROI. After that, the tonality tends to increase until it stabilizes and returns to the values initially observed, i.e., just after the air bubble passes through the ROI there is an increase of the local Hct, which tends to decrease until it stabilizes and returns to its initial values. 
From the results obtained in Fig. 4, it is possible to conclude that there is a strong influence of the air bubbles on the local Hct. These measurements show clearly that the local Hct is higher at the upstream of the bubble and lower at the downstream of the air bubble. In addition, for the $10 \%$ Hct sample, the difference between the local Hct located before and after the bubble was more evident than the difference presented in the samples with $5 \%$ Hct.

\section{Conclusions and Future Directions}

In this study, we have investigated the influence of air bubbles in the RBCs distribution when flowing through a microchannel network. The results indicate that the air bubbles strongly affect the local Hct at the regions ahead and behind them. When a bubble has approached a certain region of the microchannel, the local Hct has decreased at the downstream region. After the bubble has passed, the Hct tends to increase, and then it tends to decrease until it reaches the inlet Hct.

In the near future, we intend to verify if the difference of hematocrit before and after the bubble results only due to the method of injection of the bubble air. In addition, we also intend to study the behavior of bubbles in geometries with confluences.

Acknowledgments. This work was supported by Fundação para a Ciência e a Tecnologia (FCT), Portugal, under the strategic grants UID/EMS/04077/2019, UID/EEA/04436/2019 and UID/EMS/00532/2019. The authors are also grateful for the partial funding of FCT through the projects POCI-01-0145-FEDER-016861 (ref: PTDC/QEQ-FTT/4287/2014), NORTE-01-0145FEDER-029394, NORTE-01-0145-FEDER-030171, funded by COMPETE2020, NORTE2020, PORTUGAL2020, and FEDER. D. Bento acknowledges the PhD scholarship SFRH/BD/91192/ 2012 granted by FCT.

\section{References}

1. Muth, C.M., Shank, E.S.: Gas embolism. New Engl. J. Med. 342(7), 476-482 (2000)

2. Borger, M.A., et al.: Neuropsychologic impairment after coronary bypass surgery: effect of gaseous microemboli during perfusionist interventions. J. Thorac. cardiovasc. Surg. 121(4), 743-749 (2001)

3. Abu-Omar, Y., et al.: Solid and gaseous cerebral microembolization during off-pump, onpump, and open cardiac surgery procedures. J. Thorac. cardiovasc. Surg. 127(6), 1759-1765 (2004)

4. Bischel, M.D., Scoles, B.G., Mohler, J.G.: Evidence for pulmonary microembolization during hemodialysis. Chest 67(3), 335-337 (1975)

5. Samuel, S., et al.: In vivo microscopy of targeted vessel occlusion employing acoustic droplet vaporization. Microcirculation 19(6), 501-509 (2012)

6. Deklunder, G., et al.: Microemboli in cerebral circulation and alteration of cognitive abilities in patients with mechanical prosthetic heart valves. Stroke 29(9), 1821-1826 (1998)

7. Milo, S., et al.: Mitral mechanical heart valves: in vitro studies of their closure, vortex and microbubble formation with possible medical implications. Eur. J. Cardiothorac. Surg. 24(3), 364-370 (2003) 
8. Papadopoulou, V., et al.: A critical review of physiological bubble formation in hyperbaric decompression. Adv. Coll. Interface Sci. 191, 22-30 (2013)

9. Karlsson, L.L., et al.: Venous gas emboli and exhaled nitric oxide with simulated and actual extravehicular activity. Respir. Physiol. Neurobiol. 169, S59_S62 (2009)

10. Foster, P.P., Butler, B.D.: Decompression to altitude: assumptions, experimental evidence, and future directions. J. Appl. Physiol. 106(2), 678-690 (2009)

11. Papadopoulou, V., et al.: Circulatory bubble dynamics: from physical to biological aspects. Adv. Coll. Interface Sci. 206, 239-249 (2014)

12. Bento, D., Rodrigues, R., Faustino, V., Pinho, D., Fernandes, C., Pereira, A., Garcia, V., Miranda, J., Lima, R.: Deformation of red blood cells, air bubbles, and droplets in microfluidic devices: flow visualizations and measurements. Micromachines 9, 151 (2018)

13. Bento, D., Sousa, L., Yaginuma, T., Garcia, V., Lima, R., Miranda, J.M.: Microbubble moving in blood flow in microchannels: effect on the cell free layer and cell local concentration. Biomed. Microdevices 19(1), 6 (2017)

14. Branger, A.B., Eckmann, D.M.: Accelerated arteriolar gas embolism reabsorption by an exogenous surfactant. Anesthesiol.: J. Am. Soc. Anesthesiol. 96(4), 971-979 (2002)

15. Pinho, D., Campo-Deano, L., Lima, R., Pinho, F.T.: In vitro particulate analogue fluids for experimental studies of rheological and hemorheological behavior of glucose-rich RBCs suspensions. Biomicrofluidics 11(5), 054105 (2017) 